\title{
Identification and QTL mapping of resistance to Turnip yellows virus (TuYV) in oilseed rape, Brassica napus
}

\author{
Dieter Hackenberg ${ }^{1}$ (1) - Elvis Asare-Bediako ${ }^{1}$. Adam Baker ${ }^{1} \cdot$ Peter Walley $^{2} \cdot$ Carol Jenner $^{1} \cdot$ Shannon Greer $^{1}$. \\ Lawrence Bramham ${ }^{1}$. Jacqueline Batley ${ }^{3} \cdot$ David Edwards $^{3} \cdot$ Regine Delourme $^{4}$. Guy Barker ${ }^{1} \cdot$ Graham Teakle $^{1}$. \\ John Walsh ${ }^{1}$
}

Received: 16 August 2019 / Accepted: 24 October 2019 / Published online: 5 November 2019

(c) The Author(s) 2019

\begin{abstract}
Key message Partially dominant resistance to Turnip yellows virus associated with one major QTL was identified in the natural allotetraploid oilseed rape cultivar Yudal.

Abstract Turnip yellows virus (TuYV) is transmitted by the peach-potato aphid (Myzus persicae) and causes severe yield losses in commercial oilseed rape crops (Brassica napus). There is currently only one genetic resource for resistance to TuYV available in brassica, which was identified in the re-synthesised $B$. napus line 'R54'. In our study, 27 mostly homozygous $B$. napus accessions, either doubled-haploid (DH) or inbred lines, representing a diverse subset of the $B$. napus genepool, were screened for TuYV resistance/susceptibility. Partial resistance to TuYV was identified in the Korean spring oilseed rape, $B$. napus variety Yudal, whilst the dwarf French winter oilseed rape line Darmor- $b z h$ was susceptible. QTL mapping using the established Darmor- $b z h \times$ Yudal DH mapping population (DYDH) revealed one major QTL explaining 36\% and $18 \%$ of the phenotypic variation in two independent experiments. A DYDH line was crossed to Yudal, and reciprocal backcross $\left(\mathrm{BC}_{1}\right)$ populations from the $\mathrm{F}_{1}$ with either the susceptible or resistant parent revealed the dominant inheritance of the TuYV resistance. The QTL on ChrA04 was verified in the segregating $\mathrm{BC}_{1}$ population. A second minor QTL on ChrC05 was identified in one of the two DYDH experiments, and it was not observed in the $\mathrm{BC}_{1}$ population. The TuYV resistance QTL in 'R54' is within the QTL interval on Chr A04 of Yudal; however, the markers co-segregating with the 'R54' resistance are not conserved in Yudal, suggesting an independent origin of the TuYV resistances. This is the first report of the QTL mapping of TuYV resistance in natural B. napus.
\end{abstract}

Communicated by Albrecht E. Melchinger.

Electronic supplementary material The online version of this article (https://doi.org/10.1007/s00122-019-03469-z) contains supplementary material, which is available to authorized users.

Dieter Hackenberg

dieter.hackenberg@warwick.ac.uk

1 School of Life Sciences, University of Warwick, Wellesbourne Campus, Warwick CV35 9EF, UK

2 Functional and Comparative Genomics, Institute of Integrative Biology, University of Liverpool, Liverpool L69 7ZB, UK

3 School of Biological Sciences, Institute of Agriculture, The University of Western Australia, Crawley, WA, Australia

4 IGEPP, INRA, Agrocampus Ouest, Université Rennes, Le Rheu, France

\section{Introduction}

Oilseed rape (OSR; Brassica napus; genome AACC, $2 n=38$ ) is the second most important oilseed crop after soybean in the world; however, in Europe, OSR is the major source of vegetable oil, oilseed meals and biodiesel (Carré and Pouzet 2014). Annual global production of OSR has doubled since 2000 (FAO 2018) and reached 76.2 million tonnes in 2017 , which is equivalent to $14 \%$ of the worldwide oilseed crop production. OSR and vegetable brassicas, however, are not reaching their full yield potential in the UK. The average yield for oilseed rape in the UK (3.4 tonne/ha) (DEFRA 2018) is clearly below the estimated potential yields of current cultivars (6.5-7 tonne/ ha) (Berry and Spink 2006). Turnip yellows virus (TuYV), formerly known as Beet western yellows virus (BWYV), is considered to be a major contributor to this shortfall (Stevens et al. 2008). TuYV is a Polerovirus (Luteoviridae 
family) transmitted by aphids in a persistent, non-circulative manner. The main vector of TuYV is the peach-potato aphid Myzus persicae and annual sampling has shown that up to $72 \%$ of winged $M$. persicae carried TuYV (Stevens et al. 2008). TuYV transmission by M. persicae is highly efficient with transmission rates of over $90 \%$ (Schliephake et al. 2000). Surveys in the UK revealed that $42-70 \%$ of oilseed rape crops were infected with TuYV (Hardwick et al. 1994). Incidences of 10-85\% have been recorded within crops (Hardwick et al. 1994; Hill et al. 1989; Jay et al. 1999; Walsh et al. 1989). TuYV is seen as the most widespread and common disease in oilseed rape in Europe. Symptoms of TuYV infection are often reminiscent of abiotic stress, particularly nutrient deficiency, and can include reddening of leaves and stunted growth. In addition, TuYV infection has been shown to reduce seed yield in single OSR plants by 40-50\% (Schroeder 1994) and cause yield losses in OSR crops of 11-46\% (Graichen and Schliephake 1999; Jay et al. 1999; Jones et al. 2007). In the past, the most common strategy to control TuYV has been the use of chemical measures against the vector, in particular insecticide (neonicotinoid)-treated seeds, but most of these treatments are now banned for OSR in the $\mathrm{EU}$, emphasising the need for alternative control measures such as natural plant resistance.

The only characterised genetic source of TuYV resistance in brassica to date is the re-synthesised B. napus line 'R54'. This has been incorporated into several commercial OSR varieties. It is associated with a single dominant QTL on ChrA04 and provides incomplete resistance to TuYV (Graichen 1994; Juergens et al. 2010). The expression of the 'R54' resistance has been reported to be influenced by environmental factors such as temperature. Elevated ambient temperatures are thought to affect the development of high TuYV titres in infected plants, diminish TuYV resistance or even lead to its breakdown (Dreyer et al. 2001). A general effect of high temperature promoting stress and increasing TuYV susceptibility in OSR was reported previously (Graichen 1998). Increased TuYV incidence as a consequence of mild winters resulting in higher vector activity would indicate that global warming is likely to exacerbate yield losses of OSR crops caused by TuYV infection. Additional genetic resources for TuYV resistance breeding are gaining importance in commercial OSR production.

To identify new genetic sources of TuYV resistance, a $B$. napus diversity set representing a structured sampling of diversity across the B. napus genepool including doubled-haploid and inbred lines of winter OSR, spring OSR, kale and swede and re-synthesised B. napus, from different regions of the world were screened for TuYV susceptibility. The objective of this study was to identify QTLs associated with TuYV resistance and validate the corresponding QTLs in a $\mathrm{BC}_{1}$ generation.

\section{Materials and methods}

\section{Plant material}

Variation in TuYV susceptibility was studied in a diverse subset of 27 accessions from the B. napus diversity set developed at Warwick Crop Centre (Table 1). For QTL analysis of TuYV resistance in B. napus cultivar Yudal, two mapping populations were used. Firstly, the doubledhaploid (DH) population DYDH (Darmor- $b z h \times$ Yudal) of 120 individuals derived from the cross of French winter oilseed rape Darmor- $b z h$ line with the Korean spring oilseed rape cultivar Yudal (Delourme et al. 2006). Secondly, to verify QTLs identified in the DYDH population, a backcross $\left(\mathrm{BC}_{1}\right)$ population was produced by crossing a TuYVsusceptible DYDH line (DYDH130) to a Yudal plant to produce an $\mathrm{F}_{1}$ population (Yudal $\times$ DYDH130). Thirteen $\mathrm{F}_{1}$ plants were challenged with TuYV and tested for TuYV titre. A partially TuYV-resistant $F_{1}$ plant was crossed with the susceptible parent Darmor- $b z h$ to produce a segregating $\mathrm{BC}_{1}$ population (Darmor- $b z h \times[$ Yudal $\times \mathrm{DYDH} 130]$; see Fig. S1 for a summary of the crossing strategy). To further assess dominance/recessivity of the TuYV resistance, a second $\mathrm{BC}_{1}$ population was generated by crossing a different partially TuYV-resistant $\mathrm{F}_{1}$ plant with Yudal ([Yudal $\times$ DYDH130] $\times$ Yudal). All plants were cultivated in Levington's M2 peat compost in an insect-proof, airconditioned glasshouse at $18{ }^{\circ} \mathrm{C}$. The commercial oilseed rape variety 'Caletta' (Semundo Ltd., now Senova, Cambridge, UK) possesses the TuYV resistance originating from the re-synthesised B. napus line 'R54' and was used as a control for genotyping corresponding co-segregating markers (Juergens et al. 2010).

\section{Phenotyping}

The TuYV isolate used for phenotyping originated from OSR in Suffolk, UK (Patron 1999) and was maintained in OSR cv. 'Mikado' in an insectary under 16-h photoperiod at $20 \pm 2{ }^{\circ} \mathrm{C}$ by serial transmission using $M$. persicae (Mp1 s clone). M. persicae carrying the TuYV isolate were used to challenge plants at the 3-4 true leaf stage (3 weeks post sowing). One leaf segment from either a TuYVinfected, or from an uninfected oilseed rape plant, carrying 8-10 M. persicae, was placed on individual plants for TuYV challenge or as control, respectively. After a period of 7 days, the aphids were killed using insecticide sprays $0.4 \mathrm{ml} / \mathrm{L}$ Lambda-cyhalothrin (Hallmark Zeon, Syngenta, Fulbourn, UK) and $0.75 \mathrm{~g} / \mathrm{L}$ Pymetrozine (Plenum W.G., Syngenta, Fulbourn, UK). Two DYDH populations (SP1 and SP2; 115 DYDH lines per experiment) and two $\mathrm{BC}_{1}$ 
Table 1 Brassica napus accessions tested for resistance/ susceptibility to Turnip yellows virus (TuYV) infection

\begin{tabular}{|c|c|c|c|}
\hline Accession name & Crop type & Genetic status & Country of origin \\
\hline Apex & Winter oilseed rape & $\mathrm{S}_{1}$ & Denmark \\
\hline Bienvenu DH4 & Winter oilseed rape & DH & France \\
\hline Brauner Schnittkohl DH2 & Siberian kale & DH & Germany \\
\hline Bronowski DH1 & Spring forage rape & DH & Poland \\
\hline Canard DH13 & Winter forage rape & DH & UK \\
\hline Capricorn DH1 & Winter oilseed rape & DH & UK \\
\hline Couve Nabica DH2 & Couve nabica & DH & Portugal \\
\hline Darmor- $b z h$ & Winter oilseed rape & Inbred & France \\
\hline Dwarf Essex DH4 & Forage rape & $\mathrm{DH}$ & UK \\
\hline English Giant DH1 & Winter fodder rape & DH & UK \\
\hline Hanna DH1 & Spring oilseed rape & DH & Sweden \\
\hline Jet Neuf DH1 & Winter oilseed rape & DH & France \\
\hline Judzae DH2 & Swede landrace & DH & South Korea \\
\hline Major DH & Winter oilseed rape & DH & France \\
\hline Moana, Moana rape DH3 & Fodder rape & DH & New Zealand \\
\hline Monty-028DH & Spring OSR & DH & Australia \\
\hline Ningyou 7 & Winter oilseed rape & DH & China \\
\hline Q100 & Synthetic & DH & - \\
\hline Rafal DH1 & Winter oilseed rape & DH & France \\
\hline Sarepta DH1 & Winter OSR & DH & France \\
\hline Sensation NZ DH4 & Swede & DH & New Zealand \\
\hline Stellar DH & Spring oilseed rape & DH & Canada \\
\hline Tapidor DH & Winter oilseed rape & DH & France \\
\hline Victor & Winter oilseed rape & unspecified & Sweden \\
\hline Vige DH1 & Swede & DH & Norway \\
\hline Westar DH10 & Spring oilseed rape & DH & Canada \\
\hline Yudal & Spring oilseed rape & DH & South Korea \\
\hline
\end{tabular}

populations (200 plants per population) were challenged with TuYV. The relative levels of TuYV in plants were determined 6 weeks post-TuYV challenge by triple antibody sandwich enzyme-linked assay (TAS-ELISA) and absorbances $\left(\mathrm{A}_{405 \mathrm{~nm}}\right)$ were recorded as described previously (Hunter et al. 2002).

\section{Data analyses}

Analysis of variance and means separation using least significant differences (LSD) for TAS-ELISA $\mathrm{A}_{405 \mathrm{~nm}}$ values were carried out using GenStat Release version 12.1 (Payne et al. 2009). One-way ANOVA tests were performed to identify significant divergence between the $B$. napus accessions, D'Agostino-Pearson test for normality ( $\alpha=0.05)$ was applied to assess distribution of ELISA absorbance values in DYDH and $\mathrm{BC}_{1}$ populations used in QTL analysis experiments and non-parametric Spearman correlation was calculated to assess the reproducibility of the resistance tests of both DYDH experiments (SP1 and SP2), using GraphPad Prism version 8.1.0 (GraphPad Software, San Diego, California USA).

\section{QTL analysis}

One-hundred and twenty DH lines of the DYDH population were genotyped using a Brassica $20 \mathrm{~K}$ single nucleotide polymorphism (SNP) array (Chalhoub et al. 2014) and a genetic map was estimated using JoinMap 4 (van Ooijen 2006). For the $\mathrm{BC}_{1}$ from the Darmor- $b z h \times \mathrm{F}_{1}$ cross, DNA was isolated from 107 individuals by LGC Genomics (Hoddesdon, UK) and genotyped using the Illumina Brassica $60 \mathrm{~K}$ Infinium SNP array at the University of Western Australia. QTL associated with TuYV resistance were identified using R/qtl (Broman et al. 2003). The distribution of some ELISA values significantly deviated from normality and relevant ELISA values were transformed appropriately. If transformation did not achieve a normal distribution, single QTL analysis (scanone) was performed using a non-parametric algorithm (Kruglyak and Lander 1995). For all other datasets, single QTL (scanone) and two-dimensional QTL (scantwo) analyses were performed using the Haley-Knott regression (Haley and Knott 1992). Genome-wide LOD significance $(\alpha<0.05)$ was determined by permutation test with 10,000 permutations for scanone and 1000 for scantwo. 
For multiple QTL modelling (MQM), a stepwise forward/ backward search algorithm (stepwiseqtl; max. qtl =4) was performed to identify the QTL model of maximum penalised LOD score (Broman and Sen 2010; Manichaikul et al. 2009). Confidence intervals (CI) of 1.5 LOD were calculated and extended to the next adjacent marker to define QTL intervals.

\section{Genomic DNA extraction and PCR genotyping}

Genomic DNA was extracted from leaf tissue of B. napus accessions Yudal, Darmor-bzh and from 'Caletta' seeds according to the method published previously (Dellaporta et al. 1983). PCR amplification of the 'R54' TuYV resistance co-segregating markers was performed using specific primers for marker STS3e32m50-447-320 (forward: 5'-GAT CCGTTTGGGTCTTGGTA-3'; reverse: 5'-TTGATGTGA AACGCACATTG-3') and STS1e31 m48-437 (forward: 5'-ATCGGACATTGGTCAGGTTC-3'; reverse 5'-CATACC CCACTGGTTCTTGG-3') as described previously (Juergens et al. 2010) and amplicons were sequenced by Sanger sequencing.

\section{Results}

\section{TuYV susceptibility in diverse B. napus accessions}

The susceptibility of B. napus plant lines to TuYV varied based on virus titres determined 6 weeks after infection using TAS-ELISA. The mean virus titre (absorbance,
$\mathrm{A}_{405 \mathrm{~nm}}$ ) for the different accessions ranged from 0.485 to 1.985 (Fig. 1) with significant differences [one-way ANOVA test; $F(26,183)=1.895, p=0.0082]$. All B. napus accessions showed significantly higher mean absorbance values than the mean absorbance of the healthy control plants $\left(\mathrm{A}_{405 \mathrm{~nm}}=0.014 \pm 0.01\right)$, indicating that all tested accessions were infected by TuYV. The highest virus titre was detected in Rafal DH1 $\left(\mathrm{A}_{405 \mathrm{~nm}} \pm \mathrm{SE}=1.985 \pm 0.318\right)$, whilst Yudal had the lowest virus titre $\left(\mathrm{A}_{405 \mathrm{~nm}} \pm \mathrm{SE}=0.485 \pm 0.09\right)$. In total, 24 of the 27 B. napus accessions developed virus titres of $>50 \%$ of the maximum virus concentration measured in Rafal DH1, two accessions showed infection between 25 and $50 \%$ of the maximum and only Yudal was $<25 \%$ of the maximum. Although not showing complete resistance, Yudal $\left(\mathrm{A}_{405 \mathrm{~nm}}=0.485\right)$ had a significantly lower virus titre than all other lines (LSD for comparing mean absorbance values between accessions was 0.411 at $d f=163$, $P<0.05)$, except Sarepta $\left(\mathrm{A}_{405 \mathrm{~nm}}=0.812\right)$. The TuYV titre of challenged Darmor- $b z h$ was $>3$ times that in Yudal $\left(\mathrm{A}_{405 \mathrm{~nm}} \pm \mathrm{SE}=1.595 \pm 0.619\right.$ vs $\left.0.485 \pm 0.09\right)$, showing that Darmor- $b z h$ is significantly more susceptible to TuYV than Yudal.

\section{TuYV resistance phenotyping of Darmor-bzh $\times$ Yudal DH lines}

To map QTL for resistance in the pre-existing Darmor- $b z h$ $\times$ Yudal DH mapping population (Delourme et al. 2006), a new genetic map was generated using a B. napus $20 \mathrm{~K} \mathrm{SNP}$ array. 8152 high-quality polymorphic SNPs were identified and assembled into an initial genetic map representing the
Fig. 1 Turnip yellows virus (TuYV) challenge of Brassica napus accessions. Means and standard error of $\mathrm{A}_{405 \mathrm{~nm}}$ determined via TAS-ELISA. Yudal and Darmor- $b z h$ are indicated in white and grey, respectively

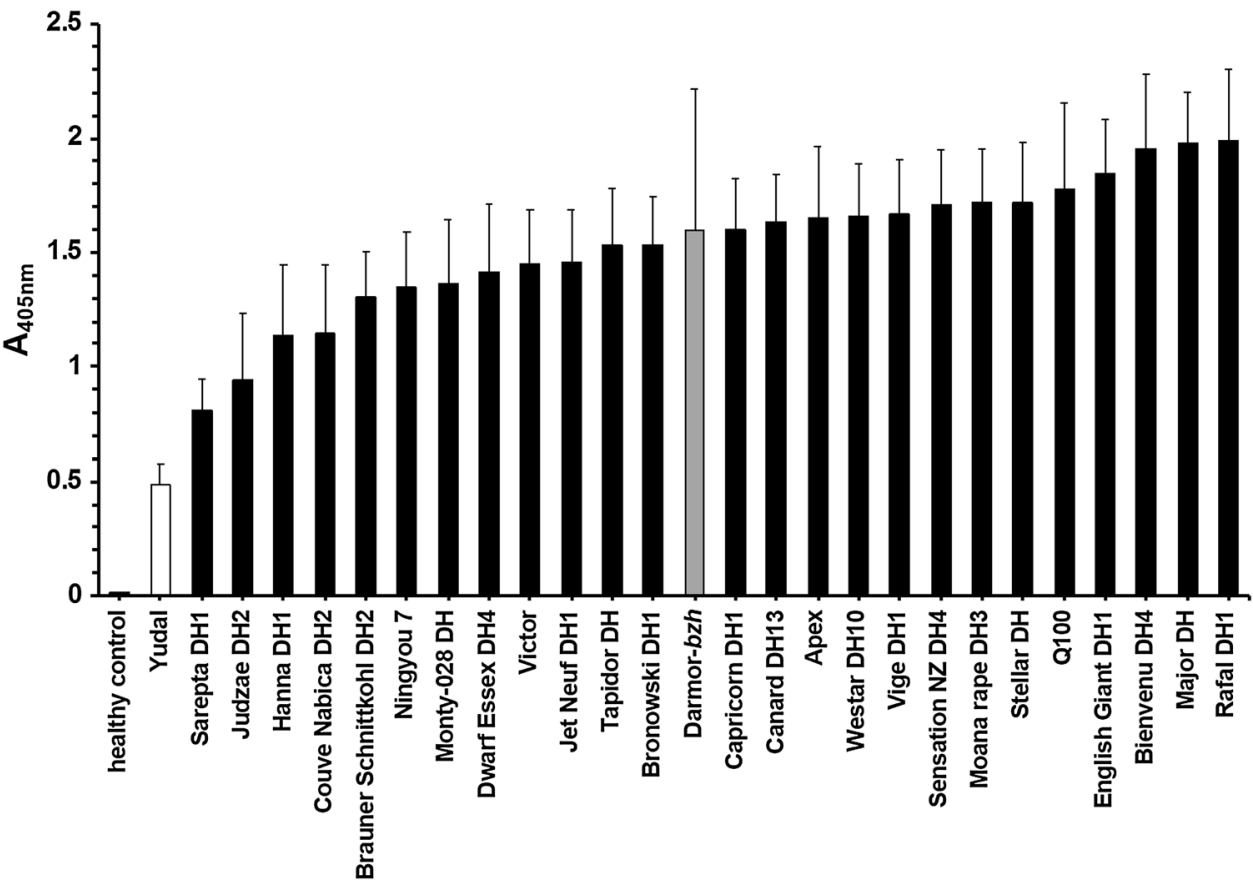


19 B. napus linkage groups. This map was simplified to a minimal map of the 1298 markers required to distinguish all the recombination events in the population and annotated according to their corresponding physical chromosomes in the B. napus genome (Chr A01 to A10 and $\mathrm{Chr} \mathrm{C} 01$ to C09). The overall size of the linkage map was $2196.3 \mathrm{cM}$ with minimum spacing of $1.7 \mathrm{cM}$ and maximum spacing of $13.3 \mathrm{cM}$. The smallest linkage group was Chr A04 (65 cM) with 39 markers and the largest was Chr C03 (196.6 cM) with 114 markers (Supp Fig. 2).

TuYV titres in 115 DYDH lines were determined in two independent experiments (SP1 and SP2). Overall mean ELISA values of both experiments were similar ( 0.62 for SP1 and 0.68 for SP2) and both test populations showed significant positive correlation in Spearman correlation test $\left(r_{s}=0.3808, P<0.0001, \alpha=0.05\right)$. Experiment SP1 and SP2 differed in their overall range of absorbance values (0.09-1.73 for SP1 and 0.27-1.18 for SP2); the majority of DH lines in SP1 and SP2 (93 and 79\%, respectively) showed mean virus titres intermediate between the resistant and susceptible parental means (Fig. 2a, b). None of the test populations showed a clear skewed or bimodal distribution

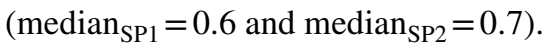

The distribution of ELISA values in SP1 (Fig. 2a) deviated significantly from a normal distribution. Appropriate transformation of SP1 ELISA values did not result in significant normality. Non-parametric QTL analysis (Fig. 2c,
Table 2) detected one significant QTL (qTUYVA4) with a LOD score of 11.3 , explaining $36.01 \%$ of the phenotypic variation at position $10 \mathrm{cM}$ on linkage group Chr A04. The QTL 1.5 LOD confidence interval (CI) was flanked by marker scaffoldv4_71_1383505 at position $0 \mathrm{cM}$ and scaffoldv4_649_37158 at position 13.9 cM, comprising a 13.9 cM QTL region (Fig. 4).

ELISA values in SP2 were normally distributed (Fig. 2b) and single QTL analysis (Fig. 2c, Table 1), multiple QTL modelling (Supp Table 1) and pairwise QTL analysis (Supp Table 2) identified two QTLs, one on Chr A04 and the other on $\mathrm{Chr} \mathrm{C05}$, which act additively, without interaction, according to the QTL model with the highest LOD score. The results for SP2 confirmed qTUYVA4 on Chr A04 as detected in $\mathrm{SP} 1$, although with a lower $\mathrm{LOD}$ score $(\mathrm{LOD}=5.11$, $\mathrm{LOD}_{\text {threshold }}=3.25 ; \alpha<0.05$ ) and a wider 1.5 LOD interval, spanning a region of $27 \mathrm{cM}$ between marker scaffoldv4_71_1383505 and scaffoldv4_455_425140 (Fig. 4). The QTL on Chr C05 $\left(\mathrm{LOD}=3.78, \mathrm{LOD}_{\text {threshold }}=3.25\right.$; $\alpha<0.05)$ explained slightly less of the phenotypic variation than that of Chr A04 (13.8\% vs. 18.2\%) and comprised a 1.5 LOD confidence interval between positions $0 \mathrm{cM}$ and $19 \mathrm{cM}$.
Fig. 2 Frequency distribution of Turnip yellows virus titres in Yudal x Darmor- $b z h$ doubled-haploid (DYDH) lines in experiments SP1 (a) and SP2 (b). Virus titres of the resistant (Yudal) and susceptible (Darmor-bzh) parents and nonlinear regression of a Gaussian distribution curve are indicated. c LOD plot of QTL analysis of DH populations SP1 experiment 1 using non-parametric interval mapping (black) and SP2 experiment 2 using Haley \& Knott (grey). LOD threshold $(\alpha \leq 0.05)$ of 10,000 permutations is indicated as a dashed line
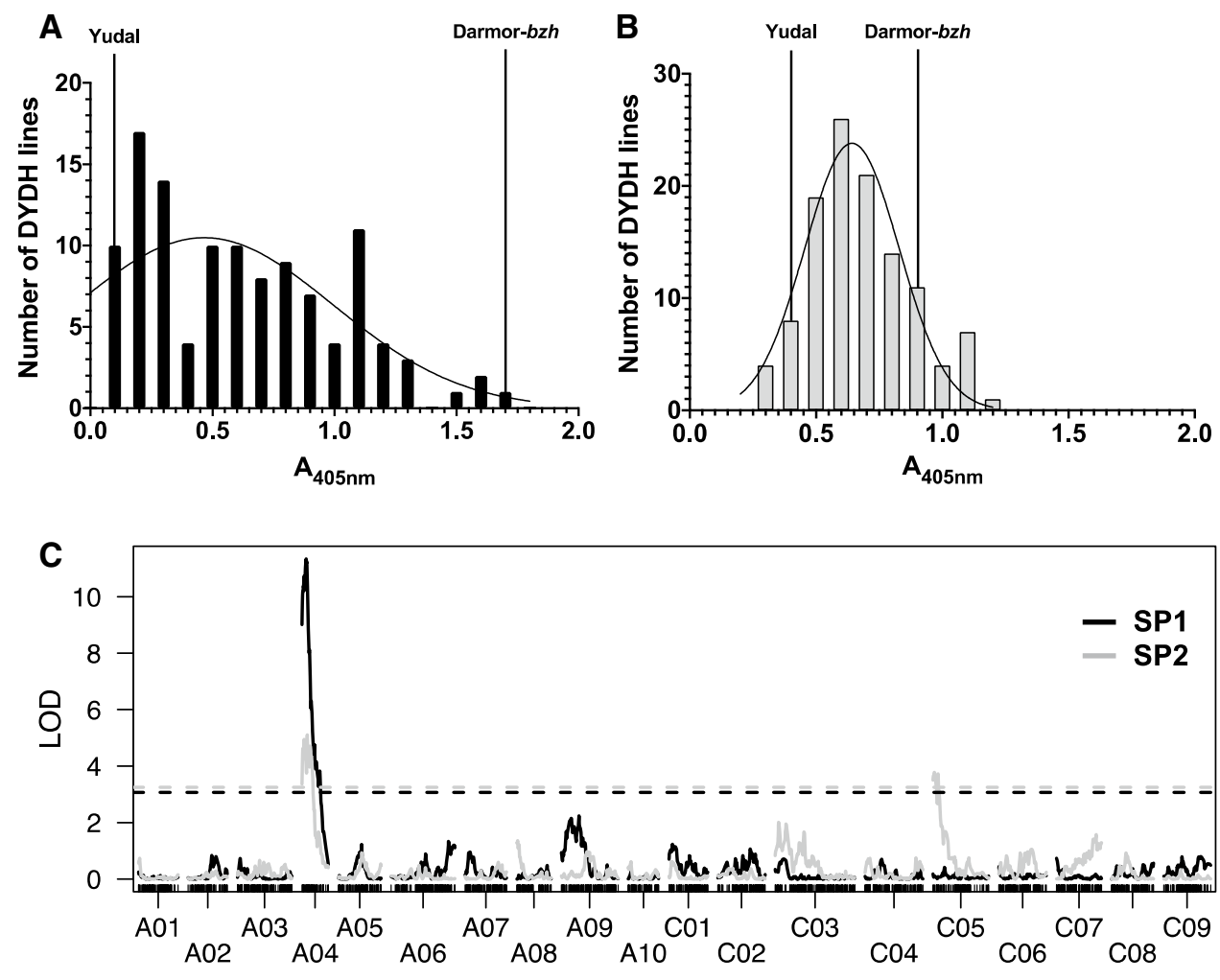

Chromosome 
Table 2 Details of quantitative trait loci (QTL) for Turnip yellows virus (TuYV) resistance detected in a BC 1 population (Darmor- $b z h \times[$ Yudal $\times$ DYDH130]) and in two experiments (SP1 and SP2) on the DYDH population

\begin{tabular}{|c|c|c|c|c|c|c|c|c|c|c|}
\hline Ex. & Name & Chr & Pos (cM) & CI & $\operatorname{LOD}(\alpha<0.05)$ & LOD & $\% R^{2}$ & Additive effect & $d f$ & Flanking markers \\
\hline SP1 & qTUYVA4 & A04 & 10 & $0-14$ & 3.07 & 11.3 & 36.0 & -0.5226 & 114 & $\begin{array}{l}\text { scaffoldv4_71_1383505 } \\
\text { scaffoldv4_649_37158 }\end{array}$ \\
\hline \multirow[t]{2}{*}{ SP2 } & qTUYVA4 & A04 & 12.9 & $0-27$ & 3.25 & 5.11 & 18.2 & -0.1637 & 114 & $\begin{array}{l}\text { scaffoldv4_71_1383505 } \\
\text { scaffoldv4_455_425140 }\end{array}$ \\
\hline & qTUYVC5 & $\mathrm{C} 05$ & 3 & 0-19 & 3.25 & 3.78 & 13.8 & 0.1388 & 114 & $\begin{array}{l}\text { scaffoldv4_266_34073 } \\
\text { scaffoldv4_215_634572 }\end{array}$ \\
\hline $\mathrm{BC}_{1}$ & qTUYVA4 & A04 & 15.5 & $0-21$ & 2.91 & 2.95 & 11.9 & -0.2318 & 106 & $\begin{array}{l}\text { Bn-A04-p560622 } \\
\text { Bn-A04-p10088142 }\end{array}$ \\
\hline
\end{tabular}

\section{QTL analysis for TuYV resistance in the $\mathrm{BC}_{1}$ population}

To determine dominance/recessivity, further investigate the potential QTL identified in the DH population and attempt to increase the mapping resolution, $\mathrm{BC}_{1}$ populations were explored. To generate a parental cross, one of the TuYVsusceptible DYDH lines (DYDH130) was crossed with Yudal (Yudal $\times$ DYDH130). The rationale for crossing a susceptible line to the resistant parent was to ensure all potential Yudal resistance alleles that would be represented in the segregating $\mathrm{BC}_{1}$ population which might not have been the case if a resistant DYDH line was crossed with Darmor- $b z h . \mathrm{F}_{1}$ plants from this cross were used to generate two $\mathrm{BC}_{1}$ populations by crossing with the susceptible Darmor- $b z h$ as female (Darmor- $b z h \times[$ Yudal $\times$ DYDH130]) and with the partially resistant Yudal as male parent ([Yudal $\times$ DYDH130] $\times$ Yudal; Supp Fig. 1). Two-hundred plants of each $\mathrm{BC}_{1}$ population were challenged with TuYV and virus titres were determined (Fig. 3a). Both $\mathrm{BC}_{1}$ populations showed a continuous distribution of ELISA values. Of the $\mathrm{BC}_{1}$ plants from the [Yudal $\times$ DYDH130] $\times$ Yudal cross, $79.5 \%$ showed ELISA values lower than the mean ELISA value of the Yudal control plants + standard deviation (threshold ${ }_{\text {Yudal+SD }}$ ). In contrast, of the $\mathrm{BC}_{1}$ plants from the Darmor- $b z h \times[$ Yudal $\times$ DYDH130] cross, $50.5 \%$ had

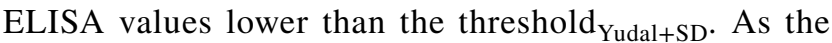
backcross to the resistant parent ([Yudal $\times$ DYDH130] $\times$ Yudal) resulted in a vast majority of $\mathrm{BC}_{1}$ partially resistant individuals, whilst the backcross with the susceptible parent (Darmor- $b z h \times[$ Yudal $\times$ DYDH130]) showed segregation of TuYV-partially resistant and TuYV -susceptible $\mathrm{BC}_{1}$ individuals, this indicated that the resistance in Yudal is dominant, but only partially dominant.

A subgroup of 107 plants of the segregating $\mathrm{BC}_{1}$ population from the Darmor- $b z h \times[$ Yudal $\times$ DYDH130] cross representing the range of the measured virus titres was genotyped for QTL analysis. Polymorphic SNPs (7790) were obtained and assembled into a minimal genetic map of 567 markers as described above for the DYDH map. Markers in this map only segregate in regions of the genome where the DYDH130 has the Darmor- $b z h$ genotype and so were not informative for QTL analysis in the DYDH130 Yudal genome segments where all the genotypes in the $\mathrm{BC}_{1}$ population would be heterozygous. The overall map size was $934.3 \mathrm{cM}$, and minimum and maximum spacing was $1.7 \mathrm{cM}$ and $18.9 \mathrm{cM}$, respectively. The linkage groups were annotated in accordance with the physical chromosomes. However, no linkage group representing ChrA05 was identified because this was entirely Yudal genotype in DYDH130, although this was not known at the time of generating the crosses. ChrA08 was annotated as two linkage groups named ChrA08A and B again because this linkage group had two Darmor- $b z h$ segments separated by a Yudal segment. The linkage groups ranged from $5.6 \mathrm{cM}$ and 6 markers (ChrA06) to $115.8 \mathrm{cM}$ and 79 markers in ChrA03 (Supp Fig. 3).

Following TuYV challenge, the mean viral titre $\left(\mathrm{A}_{405 \mathrm{~nm}}\right)$ of this $\mathrm{BC}_{1}$ population was 1.25 and ranged from 0.19 to 3.3 (Fig. 3b). The ELISA values of the Darmor- $b z h \times \mathrm{F}_{1} \mathrm{BC}_{1}$ population had to be transformed $\left(\sqrt{\mathrm{A}_{405 \mathrm{~nm}}}\right)$ to obtain normal distribution. Single QTL analysis (Fig. 3c; Table 2) and multiple QTL modelling (Supp Table 1) identified a single QTL on linkage group ChrA04, confirming qTUYVA4 in the $\mathrm{BC}_{1}$ population. The position of the QTL was determined at $15.5 \mathrm{cM}$ with a LOD score of 2.95 , slightly above the genome-wide significance threshold of 2.91 LOD $(\alpha<0.05)$, explaining $11.9 \%$ of the phenotypic variation. The QTL was linked to markers Bn-A04-p560622 at position $0 \mathrm{cM}(\mathrm{LOD}=1.50)$ and Bn-A04-p10088142 at position $21.3 \mathrm{cM}(\mathrm{LOD}=1.71)$, comprising the entire linkage group (Fig. 4). No other significant QTL was detected.

\section{Physical position of TuYV resistance in Yudal}

The physical position of the qTUYVA4 flanking markers, identified in each of the $\mathrm{DYDH}$ and $\mathrm{BC}_{1}$ experiments, was determined by sequence comparison with the $B$. napus genome of Darmor-bzh (assembly: GCA_000751015.1) 

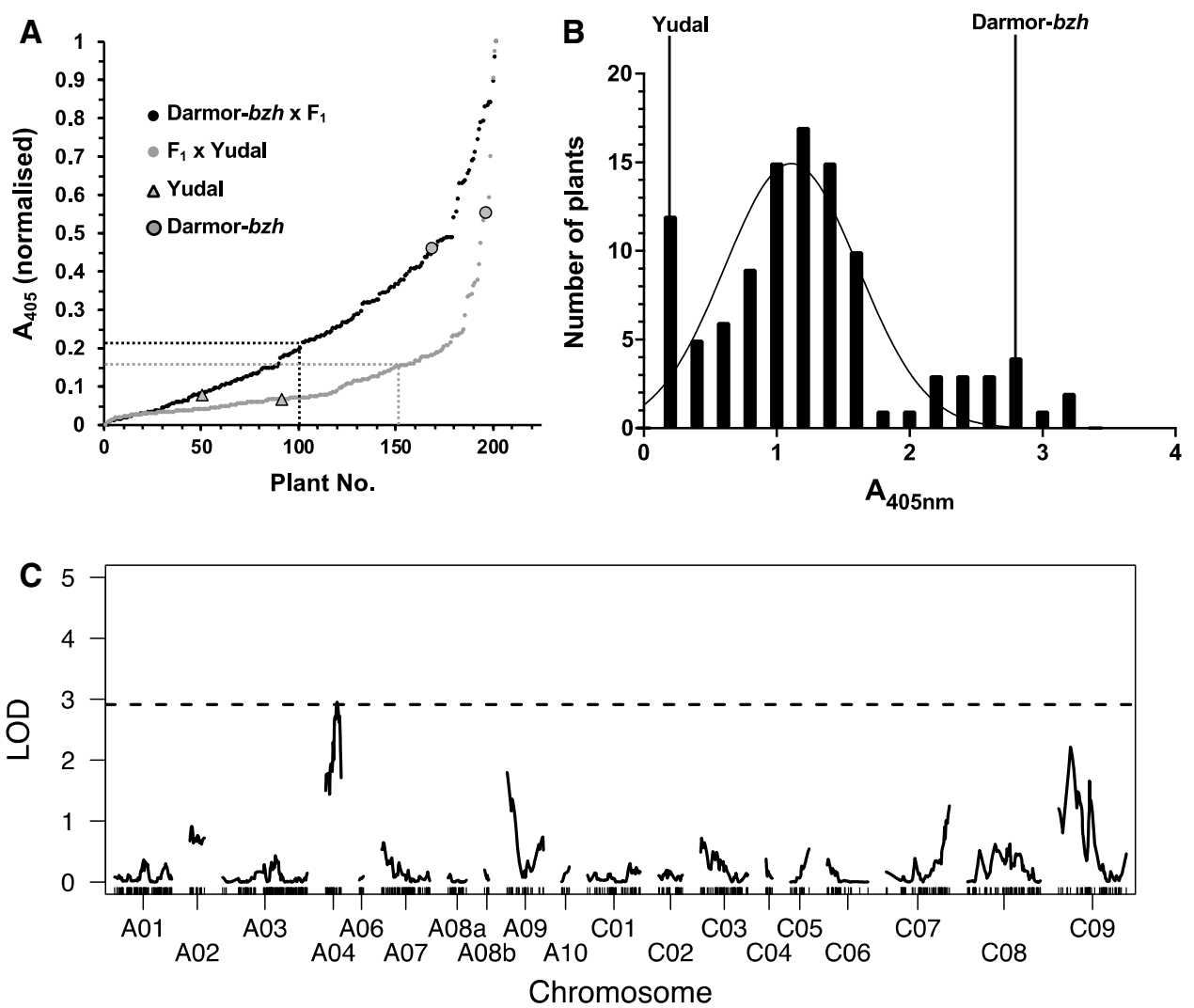

Fig. 3 a Ranked Turnip yellows virus (TuYV) titres after normalisation to the maximum and minimum TAS-ELISA absorbance values $\left(\mathrm{A}_{405}\right)$ for the $\mathrm{BC}_{1}$ populations Darmor- $b z h \times[$ Yudal $\times$ DYDH130] (black) and [Yudal $\times$ DYDH130] $\times$ Yudal (grey) together with mean values of 10-20 Yudal (triangle) and Darmor- $b z h$ (circle) control plants. The mean values for Yudal+standard deviation (threshold ${ }_{\text {Yudal+SD }}$ ) for each of the $\mathrm{BC}_{1}$ populations are indicated as

(Chalhoub et al. 2014) using the BLASTN algorithm (Altschul et al. 1990). In all the three experiments, the qTUYVA4 1.5 LOD intervals comprised overlapping regions of $\mathrm{Chr}$ A04. The smallest interval was found in experiment SP1 $(10.75 \mathrm{Mb})$ and was entirely enclosed in the larger intervals identified in the SP2 and $\mathrm{BC}_{1}$ experiments $(13.79 \mathrm{Mb}$ and $10.79 \mathrm{Mb}$ ). The minimal qTUYVA4 interval comprises 1262 annotated genes in B. napus. Since the QTL associated with TuYV resistance in 'R54' was also mapped on Chr A04, we compared its physical position with that of qTUYVA4. In the original Darmor-bzh B. napus genome assembly (Chalhoub et al. 2014), none of the co-segregating markers for the TuYV resistance in 'R54' (STS3e32m50-447-320 and STS1e31m48-437) were located on Chr A04, but could be identified on a non-annotated chromosomal scaffold (ChrAnn_random). However, both 'R54' TuYV resistance markers were unambiguously found on $\mathrm{Chr}$ A04 of the re-annotated Darmor-bzh B. napus genome assembly v8.1 (Bayer et al. 2017) and the $B$. rapa genome assembly (GCA_000309985.2) (Wang et al. 2011), encompassing a dashed line. b Frequency distribution of TuYV titres in the Darmor- $b z h \times$ [Yudal $\times \mathrm{DYDH} 130] \mathrm{BC}_{1}$ population. Mean values of 10 resistant (Yudal) and susceptible (Darmor-bzh) control plants and nonlinear regression of a Gaussian distribution curve are indicated. c LOD plot of QTL analysis of $\mathrm{BC}_{1}$ population (Darmor- $b z h \times$ [Yudal $\times$ DYDH130]) using Haley \& Knott. LOD threshold $(\alpha \leq 0.05)$ of 10,000 permutations is indicated as dashed line

approximately 3 and $4.7 \mathrm{Mb}$ regions, respectively, located inside the qTUYVA4 interval (Fig. 4). PCR analysis using the 'R54' TuYV resistance molecular markers, suggested the possible absence of the 'R54' resistance-linked alleles in Yudal and Darmor- $b z h$. Sanger sequencing of the amplicons for STS3e32m50-447-320 and STS1e31m48-437 (385 bp and $376 \mathrm{bp}$, respectively) revealed identical sequences of both markers in Yudal and Darmor- $b z h$, possessing the size of those from the susceptible plants described in the mapping of the resistance in 'R54'; STS3e32m50-447-320 was $41 \mathrm{bp}$ larger and STS1e31m48-437 was 61 bp smaller than 'R54' (Juergens et al. 2010). In contrast, the B. napus cultivar 'Caletta' (possesses the 'R54' TuYV resistance) showed homozygosity for the co-segregating alleles of the 'R54' TuYV resistance (344 bp for STS3e32m50-447-320 and $437 \mathrm{bp}$ for STS1e31 m48-437). 


\section{DYDH genetic map (cM) Physical map (Mb) $\quad$ BC $_{1}$ genetic map (cM)}

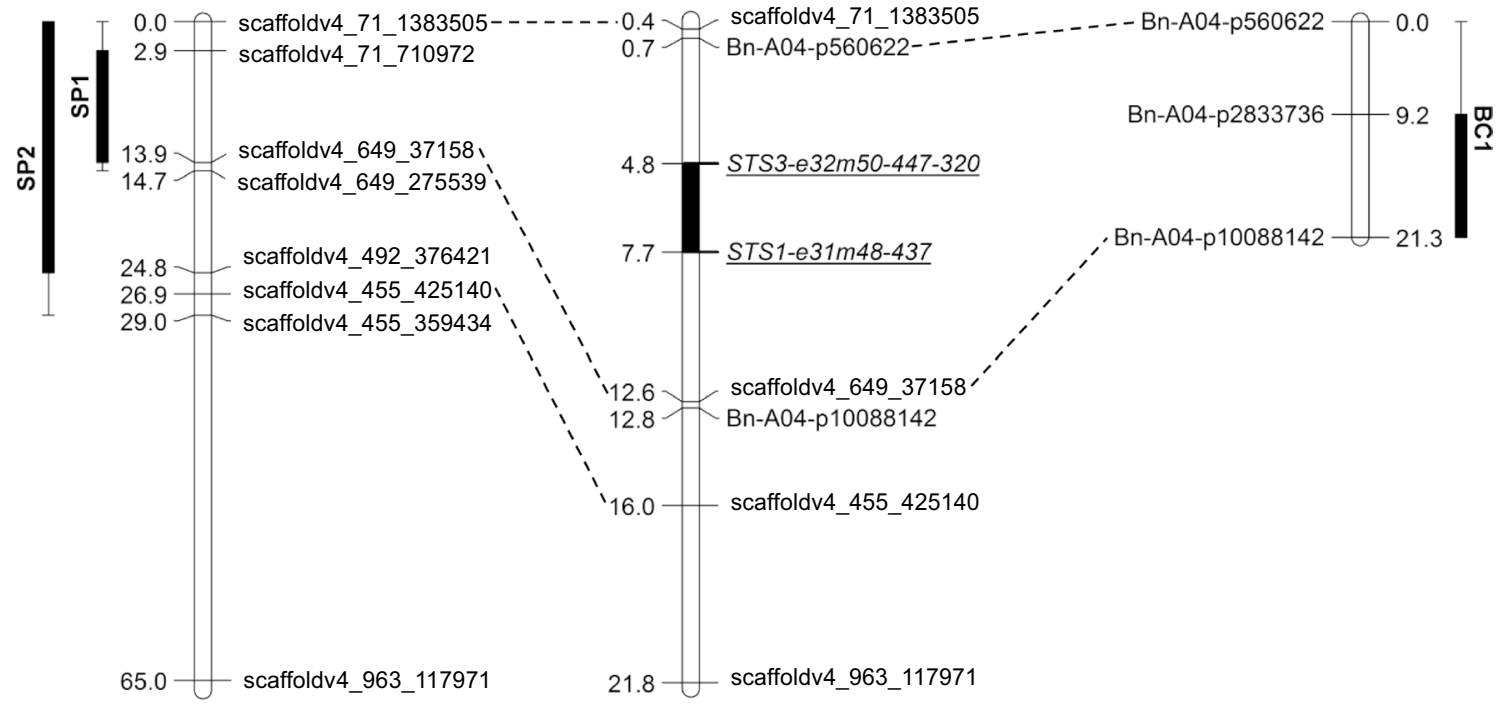

Fig. 4 Genetic linkage maps of ChrA04 from $\mathrm{DYDH}$ and $\mathrm{BC}_{1}$ experiments and physical position of Turnip yellows virus (TuYV) resistance QTL on ChrA04 using the Brassica rapa genome assembly GCA_000309985.2 v3.0

\section{Discussion}

\section{TuYV resistance in B. napus}

TuYV is one of the most widespread and common diseases, causing severe yield losses in commercial oilseed rape and vegetable brassica crops in Europe. To date, no brassica germplasm with complete immunity to TuYV has been reported. The only mapped genetic resource for TuYV resistance in brassica is the incomplete resistance in the resynthesised B. napus line 'R54' (Graichen 1994; Juergens et al. 2010), which is thought to have derived from its parental A-genome donor Chinese cabbage (Dreyer et al. 2001). In this study, we report for the first time the occurrence of TuYV resistance in a natural allotetraploid $B$. napus variety. Although the resistance was incomplete, TuYV titres in Yudal were 1.7-4.1 times lower than in any other tested accession. The resistance appears to be commercially useful, as in a controlled experiment, TuYV-challenged Yudal did not have a significantly lower seed yield than unchallenged Yudal controls, whereas other commercial varieties had significantly reduced yields (Asare-Bediako 2011).

\section{TuYV resistance inheritance and QTLs}

One major QTL for TuYV resistance in Yudal was found on ChrA04 (qTUYVA4). It was significantly associated with TuYV resistance and explained between 18 and $36 \%$ of the phenotypic variation. We determined the dominance of the TuYV resistance by phenotyping two $\mathrm{BC}_{1}$ populations.
However, the segregation of virus titres in these $\mathrm{BC}_{1}$ populations (Fig. 3a) indicated that the resistance was actually partially dominant. The qTUYVA4 interval identified in the DYDH population was verified in the segregating $\mathrm{BC}_{1}$ population, albeit with a low LOD score of only 2.95 , just passing the genome-wide significance threshold. The LOD scores for qTUYVA4 in the homozygous DYDH population were 11.3 and 5.1 in SP1 and SP2, respectively, exceeding the genomewide significance thresholds by far and the corresponding 1.5 LOD confidence interval narrowed qTUYVA4 down to 14/10.8 and 27/13.9 cM/Mb regions on Chr A04. In the segregating $\mathrm{BC}_{1}$ population, the qTUYVA4 confidence interval comprised the entire ChrA04 linkage group, providing no further resolution of qTUYVA4. This was mainly due to the weak association of qTUYVA4 with TuYV resistance and the low recombination frequency on $\mathrm{Chr} \mathrm{A} 04$ in the $\mathrm{BC}_{1}$. According to the minimal spanning tree of the $\mathrm{BC}_{1}$ genetic map, only 19 markers covered all recombination events on the $21 \mathrm{cM} / 12.8 \mathrm{Mb}$ segment of Chr A04 (Supp Fig. 3) inherited from DYDH130, representing only $58 \%$ of the physical map of Chr A04 (22.5 Mb). In contrast, the DYDH genetic map covers $21.8 \mathrm{Mb}$ of $\mathrm{Chr} \mathrm{A} 04$ generating a $65 \mathrm{cM}$ linkage group for Chr A04 and enabled mapping of qTUYVA4 between markers at positions 0.4 and $12.6 \mathrm{Mb}$ (Fig. 4). The weak association of qTUYVA4 with TuYV resistance in the $\mathrm{BC}_{1}$ population is most likely a consequence of the heterozygosity of TuYV resistance gene(s) in the $\mathrm{BC}_{1}$ and the ability to only phenotype single plants. In contrast, QTL mapping using the homozygous DH population provided higher LOD scores and resolution and facilitated phenotyping of several 
plants for each DH line in multiple experiments. This demonstrates that a DH mapping strategy was clearly advantageous for mapping this quantitative virus resistance trait.

Consistent with the nature of a quantitative trait, TuYV titres measured using the TAS-ELISA technique resulted in continuous distributions of phenotypes in all DYDH and $\mathrm{BC}_{1}$ experiments. None of the $\mathrm{DYDH}$ and $\mathrm{BC}_{1}$ phenotyping experiments showed a clear bimodal distribution of susceptible to resistant plants, as would be predicted for a 1:1 segregation of a strong monogenic trait. Thus, TuYV resistance in Yudal may depend on additional contributing genes, environmental factors and/or may be an artefact of the phenotyping of this partially dominant trait. The mapping of TuYV resistance in 'R54' showed a clear single QTL, but was based on phenotypes also not showing a bimodal distribution (Dreyer et al. 2001). Nevertheless, the markers derived from this mapping approach have been very successful in introgressing the resistance from 'R54' into the commercial oilseed rape variety 'Caletta' (Graichen and Peterka 1999). A second QTL qTUYVC5 was identified in one of the DH experiments (SP2), explaining $11.9 \%$ of the phenotypic variation, acting additively, without interaction with qTUYVA4. The estimated effects of the QTLs on Chr A04 and $\mathrm{Chr}$ C05 (Table 2) showed opposite effects of allelic substitution, suggesting that both parental lines contributed to the resistance response. Although susceptible to TuYV, Darmor- $b z h$ did not show the highest virus titre amongst the 27 tested B. napus accessions. However, as the qTUYVC5 effect was only seen in one of the two experiments on the DYDH lines, it remains speculative as to whether the slightly lower TuYV infection in Darmor-bzh was actually caused by genetic factors. No additional significant QTL were detected in either the DYDH experiment SP1 or in the segregating $\mathrm{BC}_{1}$ population, suggesting that the contribution of qTUYVC5 towards TuYV resistance may have been due to specific environmental conditions present during the SP2 experiment. The promoting effect of increased temperature on TuYV susceptibility in 'R54' and oilseed rape varieties was described previously (Dreyer et al. 2001; Graichen 1998). However, SP1 and SP2 were carried out as replicated glasshouse experiments under similar temperature regimes, but during different seasons (autumn and winter). Additional factors like light intensity or day length could have played a role during the complex interaction between plants, aphids and TuYV, influencing the course of virus infection and ultimately eliciting additional genes to contribute towards the interactions.

TuYV resistance in Yudal and in 'R54' are both associated with one major QTL, located on Chr A04. The genomic region between the two co-segregating markers of the 'R54' QTL is within the larger $10.75 \mathrm{Mb}$ CI of qTUYVA4. The 'R54' QTL and qTUYVA4 share about 166 genes. However, qTUYVA4 includes 1096 additional gene loci, which are not co-segregating with the 'R54' TuYV QTL, representing a plethora of potential alternative candidate loci for the TuYV resistance in Yudal. Intriguingly, both TuYV resistance sources are dominant and quantitative. As the co-segregating markers for the TuYV resistance in ' $\mathrm{R} 54$ ' are conserved in the commercial variety 'Caletta' (possessing resistance derived from ' $R 54$ '), but are not conserved in Yudal, this could indicate that the resistance alleles in 'R54' and qTUYVA4 are not derived from the same source. This suggests that both $B$. napus lines are very likely to have originated from different $B$. rapa A-genome donors. Yudal (B. napus var. oleifera) is a native rapeseed inbred line of Korean origin (Jeong et al. 2012; Wagner et al. 2019), developed as a high erucic acid Brassica napus cultivar in 1969 (Choy et al. 2007; Kae et al. 1971). As a native allotetraploid B. napus variety, Yudal's A-genome presumably derived from the natural hybridisation of $B$. rapa and $B$. oleracea, 5000-10,000 years ago. In contrast, 'R54' was generated via re-synthesis in the 1970s at the University of Göttingen (Juergens 2009), using a cross between the cabbage cultivar 'Stone Head' (B. oleracea var. capitata) and the Chinese cabbage 'No.67' (B. rapa ssp. pekinensis). As the underlying molecular mechanism and the gene(s) involved in the TuYV resistances in 'R54' and Yudal are unknown, it is not feasible to dismiss the possibility that both resistances are based on similar genetic loci.

TuYV infection and TuYV-related yield losses are predicted to become more severe in the future as a consequence of increasing temperature due to climate change. There are currently a number of commercial varieties of OSR with partial resistance to TuYV (for example, Amalie, Aspire, Annalise, Architect, Ambassador, Artemis, Aurelia from Limagrain, Saint-Beauzire, France; Darling, Dazzler, Ludger, Temptation from DSV, Lippstadt, Germany; Allessandro, Feliciano from KWS, Einbeck, Germany; Atora, Dominator from Rapool-Ring, Isernhagen, Germany; Cadran, Coogan from RAGT, Rodez, France; Addition from Soufflet Seeds, Poznań, Poland; DMH440 from Dekalb AgResearch, Dekalb, USA). Many of these possess resistance derived from 'R54' and no other independent TuYV resistance source is described for any of these varieties. Growing these varieties is creating strong selection pressure for resistance-breaking strains of TuYV. Additional independent sources of resistance are, therefore, essential to reduce selection pressure for 'R54' resistance-breaking TuYV and promote durable control of the virus in the future. It remains to be seen whether Yudal will provide a useful additional source of resistance to TuYV.

Acknowledgements This research was supported by the Government of Ghana Educational Trust Fund and the UK Biotechnology and Biological Sciences Research Council (BBSRC) Crop Improvement Research Club (CIRC) Project BB/I017410/1. The authors wish to thank Horticultural Services staff at the University of Warwick for 
plant care and Dr Mark Stevens, British Beet Research Organisation, Norwich for providing the TuYV isolate.

Author Contribution statement JW, EAB, CJ and GT conceived the B. napus-TuYV and DYDH experiments and EAB conducted the experiments. EAB, PW, DH, GT and LB performed the QTL analyses on the DYDH population. RD provided genotype data. GT developed the DYDH linkage map. The $\mathrm{BC}_{1}$ experiment was conceived by GB, $\mathrm{GT}, \mathrm{PW}, \mathrm{AB}$ and $\mathrm{JW}$, and $\mathrm{AB}$ performed the experiments. JB and $\mathrm{DE}$ genotyped the $\mathrm{BC}_{1}$ plants and $\mathrm{DH}, \mathrm{AB}$ and $\mathrm{GT}$ carried out the QTL analyses and developed the $\mathrm{BC}_{1}$ linkage map. SG did the ' $\mathrm{R} 54$ ' marker analysis. DH, GT and JW wrote the manuscript and all authors read and reviewed the manuscript.

Open Access This article is distributed under the terms of the Creative Commons Attribution 4.0 International License (http://creativeco mmons.org/licenses/by/4.0/), which permits unrestricted use, distribution, and reproduction in any medium, provided you give appropriate credit to the original author(s) and the source, provide a link to the Creative Commons license, and indicate if changes were made.

\section{References}

Altschul SF, Gish W, Miller W, Myers EW, Lipman DJ (1990) Basic local alignment search tool. J Mol Biol 215:403-410

Asare-Bediako E (2011) Brassicaceae-Turnip yellows virus interactions. PhD Thesis. University of Warwick

Bayer PE, Hurgobin B, Golicz AA, Chan C-KK, Yuan Y, Lee H, Renton M, Meng J, Li R, Long Y, Zou J, Bancroft I, Chalhoub B, King GJ, Batley J, Edwards D (2017) Assembly and comparison of two closely related Brassica napus genomes. Plant Biotechnol J 15:1602-1610

Berry PM, Spink JH (2006) A physiological analysis of oilseed rape yields: past and future. J Agric Sci 144:381-392

Broman KW, Sen S (2010) A guide to QTL mapping with R/qtl. Springer, New York

Broman KW, Wu H, Sen S, Churchill GA (2003) R/qtl: QTL mapping in experimental crosses. Bioinformatics 19:889-890

Carré P, Pouzet A (2014) Rapeseed market, worldwide and in Europe. Oilseeds Fats Crops Lipids 21(1):D102

Chalhoub B, Denoeud F, Liu S, Parkin IAP, Tang H, Wang X, Chiquet J, Belcram H, Tong C, Samans B, Corréa M, Da Silva C, Just J, Falentin C, Koh CS, Le Clainche I, Bernard M, Bento P, Noel B, Labadie K, Alberti A, Charles M, Arnaud D, Guo H, Daviaud C, Alamery S, Jabbari K, Zhao M, Edger PP, Chelaifa H, Tack D, Lassalle G, Mestiri I, Schnel N, Le Paslier M-C, Fan G, Renault V, Bayer PE, Golicz AA, Manoli S, Lee T-H, Thi VHD, Chalabi S, Hu Q, Fan C, Tollenaere R, Lu Y, Battail C, Shen J, Sidebottom CHD, Wang X, Canaguier A, Chauveau A, Bérard A, Deniot G, Guan M, Liu Z, Sun F, Lim YP, Lyons E, Town CD, Bancroft I, Wang X, Meng J, Ma J, Pires JC, King GJ, Brunel D, Delourme R, Renard M, Aury J-M, Adams KL, Batley J, Snowdon RJ, Tost J, Edwards D, Zhou Y, Hua W, Sharpe AG, Paterson AH, Guan C, Wincker P (2014) Early allopolyploid evolution in the postNeolithic Brassica napus oilseed genome. Science 345:950-953

Choy YH, Jeong YJ, Kim MJ, Park HM, Lee JS, Jang YS, Han IS, Yoon MS, Yoon YH (2007) Improvement of erucic acid level in Brassica napus L. Yudal. In: 12th International rapeseed conference, sustainable development in cruciferous oilseed crops production, Wuhan, China, pp 58-61
DEFRA (2018) National Statistics. Farming statistics provisional crop areas, yields and livestock populations. Department for Environment Food and Rural Affairs

Dellaporta SL, Wood J, Hicks JB (1983) A plant DNA minipreparation: version II. Plant Mol Biol Rep 1:19-21

Delourme R, Falentin C, Huteau V, Clouet V, Horvais R, Gandon B, Specel S, Hanneton L, Dheu JE, Deschamps M, Margale E, Vincourt P, Renard M (2006) Genetic control of oil content in oilseed rape (Brassica napus L.). Theor Appl Genet 113:1331-1345

Dreyer F, Graichen K, Jung C (2001) A major quantitative trait locus for resistance to Turnip Yellows Virus (TuYV, syn. beet western yellows virus, BWYV) in rapeseed. Plant Breed 120:457-462

FAO (2018) FAOSTAT statistical database. Food and Agriculture Organization of the United Nations (FAO)

Graichen K (1994) Nachweis von Resistenz gegenuber dem Turnip yellows luteovirus (TuYV) in Winterraps und verwandten. Vortraege fuer Pflanzenzuechtung 30:132-143

Graichen K (1998) Einfluß von Umweltbedingungen auf die Symptomund Resistenzauspraegung von Winterraps nach Inokulation mit dem Turnip yellows virus. Mitteilung Biologische Bundesanstalt 357:291-292

Graichen K, Peterka H (1999) Generation of oilseed rape with resistance to turnip yellows luteovirus. In: Wratten N, Salisbury PA (eds) 10th International rapeseed congress. International consultative group for rapeseed research. ACT, Canberra, pp 1-13

Graichen K, Schliephake E (1999) Infestation of winter oilseed rape by Turnip yellows virus and its effect on yield in Germany. In: 10th International rapeseed congress-new horizons from an old crop, Canberra, ACT, p 131

Haley CS, Knott SA (1992) A simple regression method for mapping quantitative trait loci in line crosses using flanking markers. Heredity (Edinb) 69:315-324

Hardwick NV, Davies JML, Wright DM (1994) The incidence of three virus diseases of winter oilseed rape in England and Wales in the 1991/92 and 1992/93 growing seasons. Plant Pathol 43:1045-1049

Hill SA, Lane A, Hardwick NV (1989) The incidence and importance of Beet western yellows virus in oilseed rape. Asp Appl Biol 23:311-318

Hunter PJ, Jones JE, Walsh JA (2002) Involvement of beet western yellows virus, cauliflower mosaic virus, and Turnip mosaic virus in internal disorders of stored white cabbage. Phytopathology 92:816-826

Jay CN, Rossall S, Smith HG (1999) Effects of beet western yellows virus on growth and yield of oilseed rape (Brassica napus). J Agric Sci 133:131-139

Jeong Y-J, Choy Y-H, Joo H-J, Hwang J-H, Byun Y-J, Ha-Lee Y-M, Lee J-S, Jang Y-S, Lee D-H (2012) Identification and analysis of cold stress-inducible genes in Korean rapeseed varieties. J Plant Biol 55:498-512

Jones RAC, Coutts BA, Hawkes J (2007) Yield-limiting potential of beet western yellows virus in Brassica napus. Aust J Agric Res $58: 788-801$

Juergens M (2009) Genetische Analyse und Kartierung einer Turnip yellows virus (TuYV)-Resistenz in Winterraps (Brassica napus L.)-Ph.D. thesis. Justus Liebig University Giessen

Juergens M, Paetsch C, Kramer I, Zahn M, Rabenstein F, Schondelmaier J, Schliephake E, Snowdon R, Friedt W, Ordon F (2010) Genetic analyses of the host-pathogen system Turnip yellows virus (TuYV)-rapeseed (Brassica napus L.) and development of molecular markers for TuYV-resistance. Theor Appl Genet 120:735-744

Kae BM, Lee JI, Kwon BS (1971) New rape variety 'Yudal'. The research report of office fo rural development. Korea Repub Nongch'on Jin Heung Chung Res Rep Crop, pp 67-70 
Kruglyak L, Lander ES (1995) A nonparametric approach for mapping quantitative trait loci. Genetics 139:1421-1428

Manichaikul A, Moon JY, Sen S, Yandell BS, Broman KW (2009) A model selection approach for the identification of quantitative trait loci in experimental crosses, allowing epistasis. Genetics 181:1077-1086

Patron N (1999) Diversity, epidemiology and the genetic control of host specificity in the genus polerovirus. Ph.D. thesis, University of East Anglia

Payne RW, Murray DA, Harding SA, Baird DB, Soutar DM (2009) Genstat for windows: introduction. VSN International, Hemel Hempstead

Schliephake E, Graichen K, Rabenstein F (2000) Investigations on the vector transmission of the Beet mild yellowing virus (BMYV) and the Turnip yellows virus (TuYV)/Untersuchungen zur Vektoruebertragung des Milden Ruebenvergilbungsvirus (Beet mild yellowing virus) und des Wasserruebenvergilbungsvirus (Turnip yellows virus). J Plant Dis Prot 107:81-87

Schroeder M (1994) Investigations on the susceptibility of oilseed rape (Brassica-napus L, $\operatorname{ssp}$ napus) to different virus diseases. J Plant Dis Prot 101:567-589

Stevens M, McGrann G, Clark B (2008) Turnip yellows virus (syn Beet western yellows virus): an emerging threat to European oilseed rape production? HGCA research review. Broom's Barn Research Centre, Higham, p 36

van Ooijen JW (2006) JoinMap®4. Software for the calculation of genetic linkage maps in experimental populations. Kyazma BV, Wageningen

Wagner G, Laperche A, Lariagon C, Marnet N, Renault D, Guitton Y, Bouchereau A, Delourme R, Manzanares-Dauleux MJ, Gravot A
(2019) Resolution of quantitative resistance to clubroot into QTLspecific metabolic modules. J Exp Bot 70:5375

Walsh JA, Perrin RM, Miller A, Laycock DS (1989) Studies on beet western yellows virus in winter oilseed rape (Brassica napus ssp. oleifera) and the effect of insecticidal treatment on its spread. Crop Prot 8:137-143

Wang X, Wang H, Wang J, Sun R, Wu J, Liu S, Bai Y, Mun J-H, Bancroft I, Cheng F, Huang S, Li X, Hua W, Wang J, Wang X, Freeling M, Pires JC, Paterson AH, Chalhoub B, Wang B, Hayward A, Sharpe AG, Park B-S, Weisshaar B, Liu B, Li B, Liu B, Tong C, Song C, Duran C, Peng C, Geng C, Koh C, Lin C, Edwards D, Mu D, Shen D, Soumpourou E, Li F, Fraser F, Conant G, Lassalle G, King GJ, Bonnema G, Tang H, Wang H, Belcram H, Zhou H, Hirakawa H, Abe H, Guo H, Wang H, Jin H, Parkin IAP, Batley J, Kim J-S, Just J, Li J, Xu J, Deng J, Kim JA, Li J, Yu J, Meng J, Wang J, Min J, Poulain J, Wang J, Hatakeyama K, Wu K, Wang L, Fang L, Trick M, Links MG, Zhao M, Jin M, Ramchiary N, Drou N, Berkman PJ, Cai Q, Huang Q, Li R, Tabata S, Cheng S, Zhang S, Zhang S, Huang S, Sato S, Sun S, Kwon S-J, Choi S-R, Lee T-H, Fan W, Zhao X, Tan X, Xu X, Wang Y, Qiu Y, Yin Y, Li Y, Du Y, Liao Y, Lim Y, Narusaka Y, Wang Y, Wang Z, Li Z, Wang Z, Xiong Z, Zhang Z (2011) The genome of the mesopolyploid crop species Brassica rapa. Nat Genet 43:1035

Publisher's Note Springer Nature remains neutral with regard to jurisdictional claims in published maps and institutional affiliations. 